\title{
Buffer Sharing in CSP-like Programs
}

\author{
Nalini Vasudevan \\ Department of Computer Science \\ Columbia University \\ New York, USA \\ naliniv@cs.columbia.edu
}

\author{
Stephen A. Edwards \\ Department of Computer Science \\ Columbia University \\ New York, USA \\ sedwards@cs.columbia.edu
}

\begin{abstract}
Most compilers focus on optimizing performance, often at the expense of memory, but efficient memory use can be just as important in constrained environments such as embedded systems.

In this paper, we present a memory reduction technique for the deterministic concurrent programming language SHIM. We focus on reducing memory consumption by sharing buffers among tasks, which use them to communicate using CSP-style rendezvous. We determine pairs of buffers that can never be in use simultaneously and use a shared region of memory for each pair.

Our technique produces a static abstraction of a SHIM program's dynamic behavior, which we then analyze to find buffers that can share memory. Experimentally, we find our technique runs quickly on modest-sized programs and can sometimes reduce memory requirements by half.
\end{abstract}

Keywords: Concurrency, SHIM, Static Analysis, Buffers, Optimization

\section{Introduction}

Embedded systems have limited memory. Overlays, which amount to time-multiplexing the use of memory regions, is one way to reduce a program's memory consumption. In this paper, we propose a technique that automatically finds opportunities to safely overlay communication buffer memory in a concurrent programming language.

The technique we present here determines what buffer memory may be shared in SHIM programs [3, 24, 25]. This is closely related to some of the techniques we have used earlier [28], although we solve a different problem.

SHIM is an asynchronous concurrent language that is scheduling-independent: its input/output behavior is not affected by any non-deterministic scheduling choices taken by its runtime environment due to processor speed, the operating system, scheduling policy, etc. A SHIM program is composed of sequential tasks that synchronize whenever they want to communicate. The language is a subset of Kahn networks [12] (to ensure determinism) that employs the rendezvous of Hoare's CSP [10] for communication to keep its behavior tractable.

SHIM processes communicate through channels. The sequence of symbols transmitted over each channel is deterministic but the relative order of symbols between channels is generally undefined. If the sequences of symbols transmitted over two channels do not interfere, we can safely share buffers. Our technique establishes ordering between pairs of channels. If we cannot find such an ordering, we conclude that the pair cannot share memory.

Our analysis is conservative: if we establish two channels can share buffers, they can do so safely, but we may miss opportunities to share certain buffers because we do not model data and may treat the program as separate pieces to avoid an exponential explosion in analysis cost. Specifically, we build sound abstractions to avoid state space explosions, effectively enumerating all possible schedules with a product machine.

One application of our technique is to minimize buffer memory used by code generated by the SHIM compiler for the Cell Broadband engine [29]. The heterogeneous Cell processor [11] consists of a power processor element (PPE) and eight synergistic processor elements (SPEs). The SHIM compiler maps tasks onto each of the SPEs. Each SPE has its own local memory and shares data through the PPE. The PPE synchronizes communication and holds all the channel buffers in its local memory. The SPE communicates with the PPE using mailboxes [13].

We wish to reduce memory used by the PPE by overlapping buffers of different channels. Our static analyzer does live range analysis on the communication channels and determines pairs of buffers that are never live at the same time. We demonstrate in Section 7 that the PPE's memory usage can be reduced drastically for practical examples such as a JPEG decoder and an FFT.

Below, we describe the SHIM language (Section 2), how we model its behavior to analyze buffer usage (Section 3), 


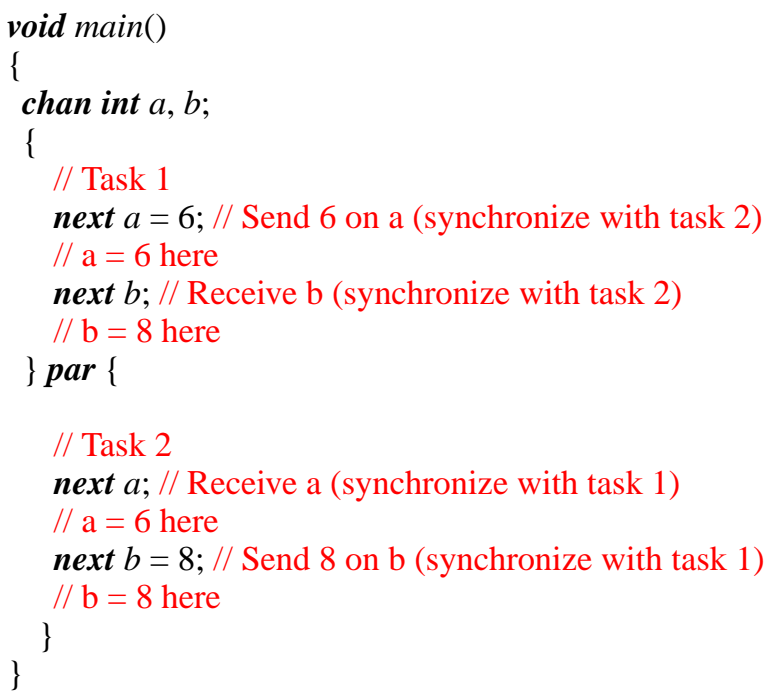

Figure 1. A SHIM program in which two tasks exchange data on channels $a$ and $b$

how we compose models of SHIM tasks to build a product machine for the whole program (Section 4), how we avoid state explosion (Section 5), and how we use these results to reduce buffer memory usage (Section 6 ). We present experimental results in Section 7 and related work in Section 8.

\section{The SHIM programming language}

SHIM [3, 24, 25] is a C-like concurrent programming language. Tasks in SHIM communicate through multi-way rendezvous channels. To the usual collection of C-like expressions and statements it adds two constructs: par for specifying concurrency and next for communication. $p$ par $q$ runs statements $p$ and $q$ in parallel and finishes when both $p$ and $q$ terminate. Next $c$ is the communication construct that synchronizes on channel $c$. Next sends data if it appears on the left side of an assignment and receives data otherwise. To ensure determinism, SHIM has no global or shared variables.

In Figure 1, two tasks run concurrently within main and communicate on channels $a$ and $b$. The next $a$ in task 1 is a send because it appears on the left side of the assignment. The next $a$ of task 2 is a receive. Similarly, the next $b$ of task 2 is a send and next $b$ of task 1 is a receive. The next $a$ in task 1 assigns 6 to $a$ and waits for task 2 to receive the value. The tasks therefore rendezvous at their nexts, then continue to the next statement. Next, the two tasks rendezvous at next $b$. There, task 1 receives the value 8 from task 2 .

The compiler rejects any program with two or more senders on a channel. If the statements next $a$ and next $b=8$ were interchanged, the program would deadlock.

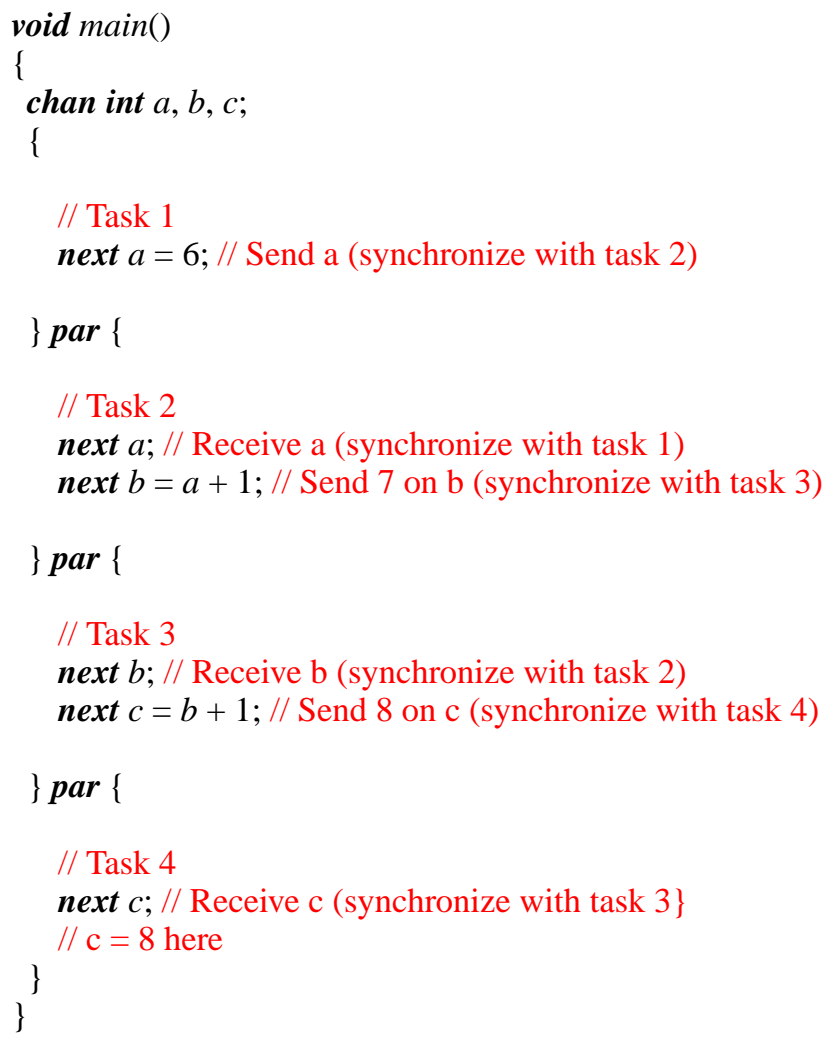

Figure 2. A SHIM program that illustrates the need for buffer sharing

SHIM can compile to C. Back ends produce code for a variety of environments: shared-memory multiprocessors using the pthreads library [6], the IBM Cell Broadband Engine [29], and single-threaded processors that do not require thread support [4]. The SHIM model has also been implemented as a library for Haskell [30]. Hardware translation has also been proposed [5] but has not yet been implemented.

In this paper we address an optimizing technique for SHIM: buffer sharing. In the program in Figure 2, the main task starts four tasks in parallel. Tasks 1 and 2 communicate on $a$. Then, tasks 2 and 3 communicate on $b$ and finally tasks 3 and 4 on $c$. The value of $c$ received by task 4 is 8 . Communication on $a$ cannot occur simultaneously with that of $b$ because task 2 forces them to occur sequentially them. Similarly communications on $b$ and $c$ are forced to be sequential by task 3 . Communications on $a$ and $c$ cannot occur together because they are forced to be sequential by the communication on $b$. Our tool understands this pattern and reports that $a, b$, and $c$ can share buffers because their communications never overlap, thereby reducing the total buffer requirements by $66 \%$ for this program. 


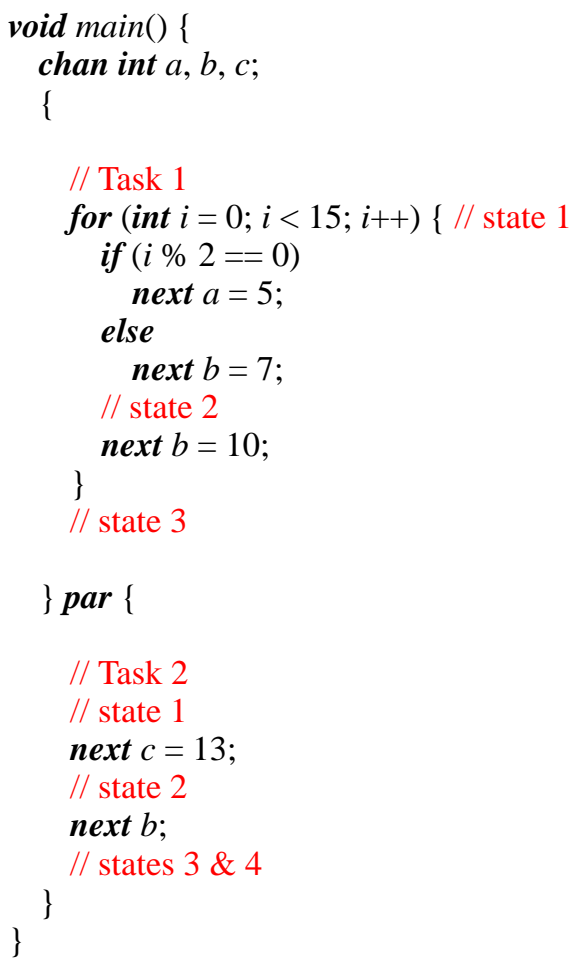

Figure 3. A (contrived) SHIM program with a loop, conditionals, and a task that terminates

\section{Abstracting SHIM Programs}

First, we assume that a SHIM program has no recursion. We use the techniques of Edwards and Zeng [7] to remove bounded recursion, which makes the program finite and renders the buffer minimization problem decidable. We do not attempt to analyze programs with unbounded recursion.

Although the recursion-free subset of SHIM is finitestate and therefore tractable in theory, in practice the full state space of even a small program is usually too large to analyze exactly; a sound abstraction is necessary. A SHIM task has both computation and communication, but because buffers are used only when tasks communicate, we abstract away the computation.

Since we abstract away computation, we must assume that all branches of any conditional statement can be taken. This leaves open the possibility that two channels may appear to be used simultaneously but in fact never are, but we believe our abstraction is reasonable. In particular it is safe: we overlap buffers only when we are sure that two channels can never be used at the same time regardless of the details of the computation.

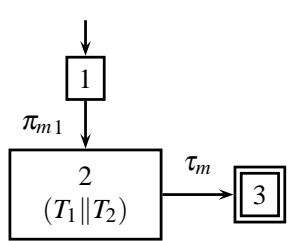

(a) $M\left(\mathrm{a}, \mathrm{b}, \mathrm{c}, \pi_{m 1}\right)$

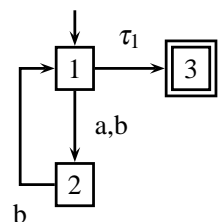

(b) $T_{1}\left(\mathrm{a}, \mathrm{b}, \tau_{1}\right)$

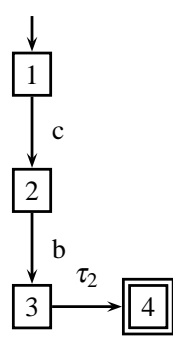

(c) $T_{2}\left(\mathrm{~b}, \mathrm{c}, \tau_{2}\right)$
Figure 4. The main task and its subtasks

\subsection{An Example}

In Figure 3, the main function consists of two tasks that communicate through channels $a, b$, and $c$.

The first task communicates on channels $a$ and $b$ in a loop; the second task synchronizes on channels $c$ and $b$, then terminates. Once a task terminates, it is no longer compelled to synchronize on the channels to which it is connected. Thus after the second task terminates, the first task just talks to itself, i.e., it is the only process that participates in a rendezvous on its channels. Terminated processes do not cause other processes to deadlock.

At compilation time, the compiler dismantles the main function of Figure 3 into tasks $T_{1}$ and $T_{2} . T_{1}$ is connected to channels $a$ and $b$ since a and $\mathrm{b}$ appear in the code section of $T_{1}$. Similarly $T_{2}$ is connected to channels $b$ and $c$. During the first iteration of the loop in $T_{1}, T_{1}$ talks to itself on $a$; since no other task is connected to $a$. Meanwhile, $T_{2}$ talks to itself on $c$. Then the two tasks rendezvous on $b$, communicating the value 10 , then $T_{2}$ terminates. During subsequent iterations of $T_{1}, T_{1}$ talks to itself on either $b$ twice or $a$ and $b$ once each.

In the program in Figure 3, communication on $b$ cannot occur simultaneously with that on c because $T_{2}$ forces the two communications to be sequential and therefore $b$ and $c$ can share buffers. On the other hand, there is no ordering between channels $a$ and $c ; a$ and $c$ can rendezvous at the same time and therefore $a$ and $c$ cannot share buffers. By overlapping the buffers of $b$ and $c$, we can save $33 \%$ of the total buffer space.

Our analysis performs the same preprocessing as our static deadlock detector [28]. It begins by removing bounded recursion using Edwards and Zeng's technique [7]. Next, we duplicate functions to force every call site to be unique. This has the potential of producing an exponential blow-up, but we have not observed it in practice.

At this point, the call graph of the program is a tree, enabling us to statically determine all the tasks and the channels to which each is connected. 
Next we disregard all functions that do not affect the communication behavior of the program. Because we are ignoring data, their behavior cannot affect whether we consider a buffer to be sharable. We implicitly assume every such function can terminate-again, a safe approximation.

Next, we create an automaton that models the control and communication behavior for each function. Figure 4 shows automata for the three tasks (main, $T_{1}$, and $T_{2}$ ) of Figure 3. For each task, we build a deterministic finite state automaton whose edges represent choices, typically to communicate. The states are labeled by program counter values and the transitions by channel names. Each automaton has a unique final state, which we draw as a double box. There is a transition from every terminating state to this final state labeled with a dummy channel that indicates such a transition. An automaton has only one final state but can have multiple terminating states. In the $T_{1}$ of Figure 3 , state 1 is the terminating state, state 3 is the final state, and they are connected by $\tau_{1}$, which is like a classical $\varepsilon$ transition. However, a true $\varepsilon$ transition would make the automaton non-deterministic, so we instead create the dummy channel $\tau_{1}$ that is unique to $T_{1}$ and allow $T_{1}$ to freely move from state 1 to state 3 without having to synchronize with any other another task.

The main function has a dummy $\pi_{m 1}$ transition from its start to the entry of state $2\left(T_{1} \| T_{2}\right)$, which represents the par statement in main. In general, we create a dummy channel for every par in the program.

Figure 5(a) shows the product of $T_{1}$ and $T_{2}$-an automaton that represents the combined behavior of $T_{1}$ and $T_{2}$. We constructed Figure 5(a) as follows. We start with state (program counter) values $(1,1)$. At this point, $T_{1}$ can communicate on $a$ and move to state 2 . Therefore we have an arc from $(1,1)$ to $(2,1)$ labeled $a$. Similarly, $T_{2}$ can communicate on $c$ and move to its state 2 . From state $(1,1)$ it is not possible to communicate on $b$ because only $T_{1}$ is ready to communicate, not $T_{2}\left(T_{2}\right.$ is also connected to $\left.b\right)$. Also at state $(1,1), T_{1}$ can terminate by taking the transition $\tau_{1}$ and moving to $(3,1)$.

From state $(3,1), T_{2}$ can transition first to state $(3,2)$ by communicating on channel $c$ and then to state $(3,3)$ by communicating on $b$; these transitions do not change the state of $T_{1}$ because it has already terminated.

From $(2,1), T_{2}$ can communicate on $c$ and change the state to $(2,2)$. Similarly from $(1,2), T_{1}$ can communicate on $a$ and move to $(2,2)$. In state $(1,2)$ it is also possible to communicate on $b$ since both tasks are ready. Therefore, we have an arc $b$ from $(1,2)$ to $(2,3)$. Since $T_{1}$ may also choose to terminate in state $(1,2)$, there is an arc from $(1,2)$ to $(3,2)$ on $\tau_{1}$. Other states follow similar rules.

To determine which channels may share buffers, we consider all states that have two or more outgoing edges. For example, in Figure 5(a), state $(1,1)$ has outgoing transitions on $a$ and $c$. Either of them can fire, so this is a case where the program may choose to communicate on either $a$ or $c$. This means the contents of both of these buffers are needed at this point, so we conclude buffers for $a$ and $c$ may not share memory. We prove this formally later in the paper.

From Figure 3, it is evident that $a$ and $b$ can never occur together because $T_{1}$ forces them to be sequential. However, since state $(1,2)$ has outgoing transitions on $a$ and $b$, our algorithm concludes that $a$ and $b$ can occur together. However, they actually can not. We draw this erroneous conclusion because our algorithm does not differentiate between scheduling choices and control flow choices (i.e., due to conditionals such as if and while). By doing this we are only adding extra behavior to the system and disregarding pairs of channels whose buffers actually could be shared. This is not a big disadvantage because our analysis remains safe. For this example, our algorithm only allows $b$ and $c$ to share buffers.

Figure 5(b) is obtained by inlining the automaton for $T_{1} \cdot T_{2}$-Figure 5(a)—within $M$. This represents the entire program in Figure 3. Since the par call is blocking, inlining $T_{1} \cdot T_{2}$ within $M$ is safe. We replaced state 2 of Figure 4(a) with Figure 5(a) to obtain Figure 5(b). The conclusions are the same as that of Figure 5(a) - only $b$ and $c$ can share buffers.

\section{Merging Tasks}

In this section, we use notation from automata theory to formalize the merging of two tasks. We show our algorithm does not generate any false negatives and is therefore safe.

Definition 1 A deterministic finite automaton $T$ is a 5-tuple $(Q, \Sigma, \delta, q, f)$ where $Q$ is the set of states, $\Sigma$ is the set of channels, $q \in Q_{1}$ is the initial state, $f \in Q$ is the final state, and $\delta \subseteq Q \times \Sigma \rightarrow Q$ is the partial transition function.

Definition 2 If $T_{1}$ and $T_{2}$ are automata, then the composed automaton $T_{1} \cdot T_{2}=\left(Q_{1} \times Q_{2}, \Sigma_{1} \cup \Sigma_{2}, \delta_{12},\left\langle q_{1}, q_{2}\right\rangle,\left\langle f_{1}, f_{2}\right\rangle\right)$, where, for $\left\langle p_{1}, p_{2}\right\rangle \in Q_{1} \times Q_{2}$ and $a \in \Sigma_{1} \cup \Sigma_{2}$,

$$
\delta_{12}\left(\left\langle p_{1}, p_{2}\right\rangle, a\right)=\left\{\begin{array}{cl}
\left\langle\delta_{1}\left(p_{1}, a\right),\right. & \text { if } a \in \Sigma_{1} \text { and } a \in \Sigma_{2} \\
\left.\delta_{2}\left(p_{2}, a\right)\right\rangle & \\
\left\langle\delta_{1}\left(p_{1}, a\right), p_{2}\right\rangle & \text { if } a \in \Sigma_{1} \text { and } \\
& \left(a \notin \sum_{2} \text { or } p_{2}=f_{2}\right) ; \\
\left\langle p_{1}, \delta_{2}\left(p_{2}, a\right)\right\rangle & \text { if } a \in \Sigma_{2} \text { and } \\
& \left(a \notin \sum_{1} \text { or } p_{1}=f_{1}\right) ; \\
\text { undefined } & \text { otherwise; }
\end{array}\right.
$$

is the transition rule for composition.

In general, if $T_{1}$ has $m$ states and $T_{2}$ has $n$, then the product $T_{1} \cdot T_{2}$ can have at most $m n$ states. The states are labeled by a tuple composed of the program counter values of 


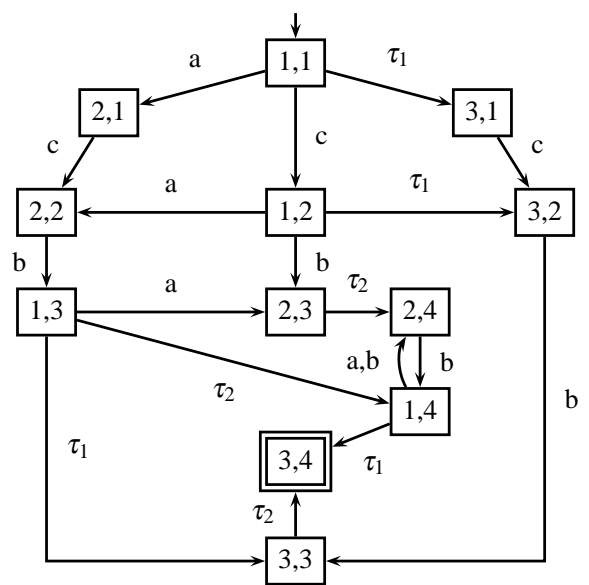

(a) $T_{1} \cdot T_{2}\left(\mathrm{a}, \mathrm{b}, \mathrm{c}, \tau_{1}, \tau_{2}\right)$

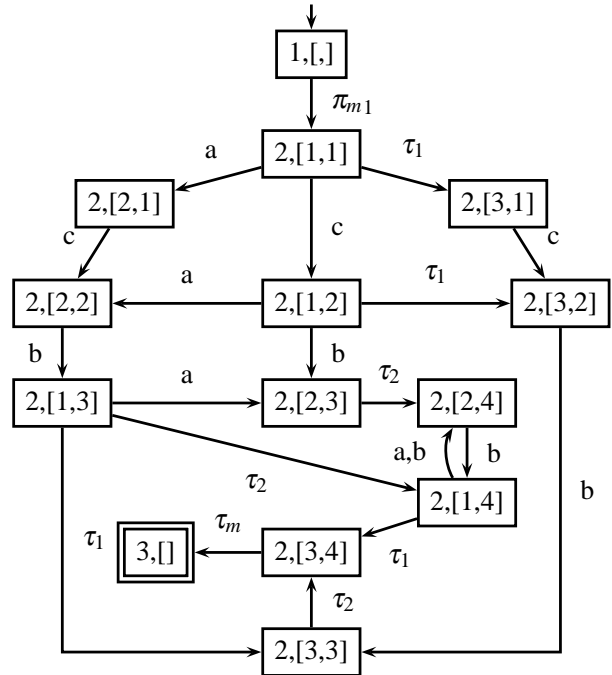

(b) $M \cdot T_{1} \cdot T_{2}\left(\mathrm{a}, \mathrm{b}, \mathrm{c}, \tau_{1}, \tau_{2}, \pi_{m 1}\right)$

Figure 5. Composing tasks in Figure 4: (a) Merging $T_{1}$ and $T_{2}$. (b) Inlining $T_{1} \cdot T_{2}$ in $M$.

the individual tasks. Each state can have at most $k$ outgoing edges, where $k$ is the total number of channels. Consequently, the total number of edges in the graph can at most be $m n k$ ( $k$ accounts for the extra $\tau$ and $\pi$ channels-one extra channel per task and one per par).

Below, we demonstrate that the order in which automata are composed does not matter. Although the state labels will be different, the states are isomorphic. First, we define exactly what we mean for two automata to be equivalent.

Definition 3 Two automata $T_{1}=\left(Q_{1}, \Sigma_{1}, \delta_{1}, q_{1}, f_{1}\right)$ and $T_{2}=\left(Q_{2}, \Sigma_{2}, \delta_{2}, q_{2}, f_{2}\right)$ are equivalent (written $\left.T_{1} \equiv T_{2}\right)$ if and only if $\Sigma_{1}=\Sigma_{2}$ and there exists a bijective function $b: Q_{1} \rightarrow Q_{2}$ such that $q_{2}=b\left(q_{1}\right), f_{2}=b\left(f_{1}\right)$, and for every $p \in Q_{1}$ and $a \in \Sigma_{1}$, either both $\delta_{1}(p, a)$ and $\delta_{2}(b(p), a)$ are defined and $\delta_{2}(b(p), a)=b\left(\delta_{1}(p, a)\right)$ or both are undefined.

Lemma 1 Composition is commutative: $T_{1} \cdot T_{2} \equiv T_{2} \cdot T_{1}$.

PROOF By definition,

$$
\begin{aligned}
& T_{1} \cdot T_{2}=\left(Q_{1} \times Q_{2}, \Sigma_{1} \cup \Sigma_{2}, \delta_{12},\left\langle q_{1}, q_{2}\right\rangle,\left\langle f_{1}, f_{2}\right\rangle\right) \text { and } \\
& T_{2} \cdot T_{1}=\left(Q_{2} \times Q_{1}, \Sigma_{2} \cup \Sigma_{1}, \delta_{21},\left\langle q_{2}, q_{1}\right\rangle,\left\langle f_{2}, f_{1}\right\rangle\right) .
\end{aligned}
$$

We claim $b\left(\left\langle p_{1}, p_{2}\right\rangle\right)=\left\langle p_{2}, p_{1}\right\rangle$ is a suitable bijective function. First, note $\Sigma_{1} \cup \Sigma_{2}=\Sigma_{2} \cup \Sigma_{1},\left\langle q_{2}, q_{1}\right\rangle=b\left(\left\langle q_{1}, q_{2}\right\rangle\right)$, and $\left\langle f_{2}, f_{1}\right\rangle=b\left(\left\langle f_{1}, f_{2}\right\rangle\right)$.
Next,

$$
\begin{aligned}
& \delta_{21}\left(b\left(\left\langle p_{1}, p_{2}\right\rangle\right), a\right) \\
& =\delta_{21}\left(\left\langle p_{2}, p_{1}\right\rangle, a\right) \\
& = \begin{cases}\left\langle\delta_{2}\left(p_{2}, a\right), \delta_{1}\left(p_{1}, a\right)\right\rangle & \text { if } a \in \Sigma_{2} \text { and } a \in \Sigma_{1} ; \\
\left\langle\delta_{2}\left(p_{2}, a\right), p_{1}\right\rangle & \text { if } a \in \Sigma_{2} \text { and } \\
\left\langle p_{2}, \delta_{1}\left(p_{1}, a\right)\right\rangle & \left(a \notin \Sigma_{1} \text { or } p_{1}=f_{1}\right) ; \\
& \text { if } a \in \Sigma_{1} \text { and } \\
\text { undefined } & \left(a \notin \Sigma_{2} \text { or } p_{2}=f_{2}\right) ;\end{cases} \\
& =b\left(\begin{array}{ll}
\left\langle\delta_{1}\left(p_{1}, a\right), \delta_{2}\left(p_{2}, a\right)\right\rangle & \text { if } a \in \Sigma_{1} \text { and } a \in \Sigma_{2} ; \\
\left\langle p_{1}, \delta_{2}\left(p_{2}, a\right)\right\rangle & \text { if } a \in \Sigma_{2} \text { and } \\
& \left(a \notin \Sigma_{1} \text { or } p_{1}=f_{1}\right) ; \\
\left\langle\delta_{1}\left(p_{1}, a\right), p_{2}\right\rangle & \text { if } a \in \Sigma_{1} \text { and } \\
& \left(a \notin \Sigma_{2} \text { or } p_{2}=f_{2}\right) ; \\
\text { undefined } & \text { otherwise; }
\end{array}\right) \\
& =b\left(\delta_{12}\left(\left\langle p_{1}, p_{2}\right\rangle, a\right)\right)
\end{aligned}
$$

Thus, $T_{1} \cdot T_{2} \equiv T_{2} \cdot T_{1}$.

Lemma 2 Composition is associative: $\left(T_{1} \cdot T_{2}\right) \cdot T_{3} \equiv T_{1}$. $\left(T_{2} \cdot T_{3}\right)$.

PROOF By definition,

$$
\begin{aligned}
\left(T_{1} \cdot T_{2}\right) \cdot T_{3}= & \left(\left(Q_{1} \times Q_{2}\right) \times Q_{3},\left(\Sigma_{1} \cup \Sigma_{2}\right) \cup \Sigma_{3}, \delta_{(12) 3},\right. \\
& \left.\left\langle\left\langle q_{1}, q_{2}\right\rangle, q_{3}\right\rangle,\left\langle\left\langle f_{1}, f_{2},\right\rangle, f_{3}\right\rangle\right) \\
T_{1} \cdot\left(T_{2} \cdot T_{3}\right)= & \left(Q_{1} \times\left(Q_{2} \times Q_{3}\right), \Sigma_{1} \cup\left(\Sigma_{2} \cup \Sigma_{3}\right), \delta_{1(23)},\right. \\
& \left.\left\langle q_{1},\left\langle q_{2}, q_{3}\right\rangle\right\rangle,\left\langle f_{1},\left\langle f_{2}, f_{3}\right\rangle\right\rangle\right) .
\end{aligned}
$$


We claim $b\left(\left\langle\left\langle p_{1}, p_{2}\right\rangle, p_{3}\right\rangle\right)=\left\langle p_{1},\left\langle p_{2}, p_{3}\right\rangle\right\rangle$ is a suitable bijective function. First, note that $\left(\Sigma_{1} \cup \Sigma_{2}\right) \cup$ $\Sigma_{3}=\Sigma_{1} \cup\left(\Sigma_{2} \cup \Sigma_{3}\right),\left\langle q_{1},\left\langle q_{2}, q_{3}\right\rangle\right\rangle=b\left(\left\langle\left\langle q_{1}, q_{2}\right\rangle, q_{3}\right\rangle\right)$, and $\left\langle f_{1},\left\langle f_{2}, f_{3}\right\rangle\right\rangle=b\left(\left\langle\left\langle f_{1}, f_{2}\right\rangle, f_{3}\right\rangle\right)$.

Next,

$$
\begin{aligned}
& \delta_{1(23)}\left(b\left(\left\langle\left\langle p_{1}, p_{2}\right\rangle, p_{3}\right\rangle\right), a\right) \\
& =\delta_{1(23)}\left(\left\langle p_{1},\left\langle p_{2}, p_{3}\right\rangle\right\rangle, a\right) \\
& \left\{\begin{array}{cl}
\left\langle\delta_{1}\left(p_{1}, a\right),\left\langle\delta_{2}\left(p_{2}, a\right),\right.\right. & \text { if } a \in \sum_{1} \text { and } a \in \sum_{2} \text { and } \\
\left.\left.\delta_{3}\left(p_{3}, a\right)\right\rangle\right\rangle & a \in \sum_{3} ; \\
\left\langle\delta_{1}\left(p_{1}, a\right),\left\langle\delta_{2}\left(p_{2}, a\right), p_{3}\right\rangle\right\rangle & \text { if } a \in \sum_{1} \text { and } a \in \sum_{2} \text { and } \\
& \left(a \notin \sum_{3} \text { or } p_{3}=f_{3}\right) ; \\
\left\langle\delta_{1}\left(p_{1}, a\right),\left\langle p_{2}, \delta_{3}\left(p_{3}, a\right)\right\rangle\right\rangle & \text { if } a \in \sum_{1} \text { and } a \in \sum_{3} \text { and } \\
& \left(a \notin \sum_{2} \text { or } p_{2}=f_{2}\right) ; \\
\left\langle\delta_{1}\left(p_{1}, a\right),\left\langle p_{2}, p_{3}\right\rangle\right\rangle & \text { if } a \in \sum_{1} \text { and } \\
& \left(a \notin \sum_{2} \text { or } p_{2}=f_{2}\right) \text { and } \\
& \left(a \notin \sum_{3} \text { or } p_{3}=f_{3}\right) ; \\
\left\langle p_{1},\left\langle\delta_{2}\left(p_{2}, a\right), \delta_{3}\left(p_{3}, a\right)\right\rangle\right\rangle & \text { if } a \in \sum_{2} \text { and } a \in \sum_{3} \text { and } \\
\left\langle p_{1},\left\langle\delta_{2}\left(p_{2}, a\right), p_{3}\right\rangle\right\rangle & \left(a \notin \sum_{1} \text { or } p_{1}=f_{1}\right) ; \\
& \text { if } a \in \sum_{2} \text { and } \\
& \left(a \notin \sum_{1} \text { or } p_{1}=f_{1}\right) \text { and } \\
\left\langle p_{1},\left\langle p_{2}, \delta_{3}\left(p_{3}, a\right)\right\rangle\right\rangle & \left(a \notin \sum_{3} \text { or } p_{3}=f_{3}\right) ; \\
& \text { if } a \in \sum_{3} \text { and } \\
& \left(a \notin \sum_{1} \text { or } p_{1}=f_{1}\right) \text { and } \\
\text { undefined } & \left(a \notin \sum_{2} \text { or } p_{2}=f_{2}\right) ; \\
& \text { otherwise; }
\end{array}\right.
\end{aligned}
$$

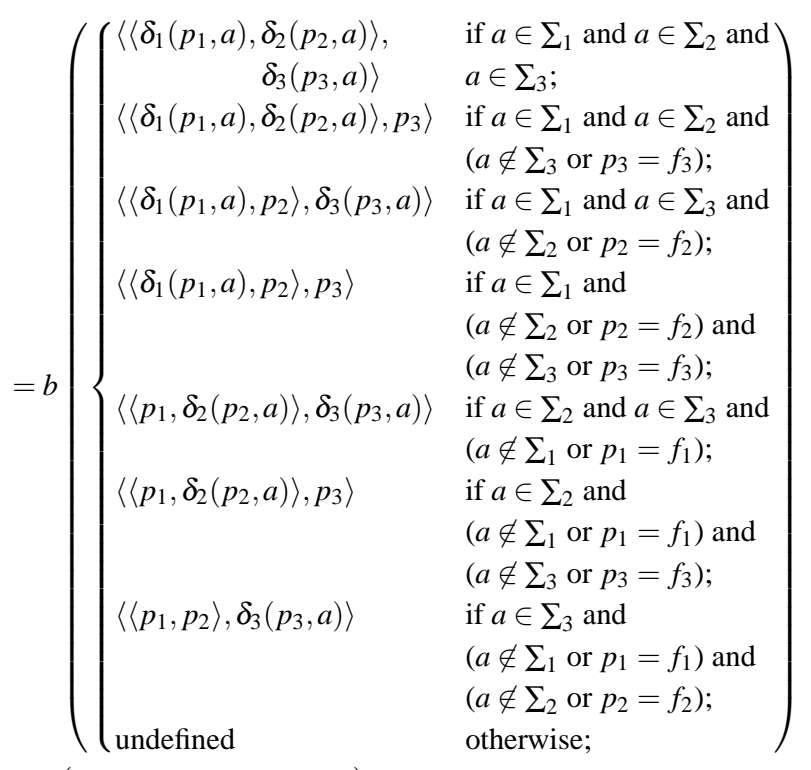$$
=b\left(\delta_{(12) 3}\left(\left\langle\left\langle p_{1}, p_{2}\right\rangle, p_{3}\right\rangle, a\right)\right)
$$

Thus, $\left(T_{1} \cdot T_{2}\right) \cdot T_{3} \equiv T_{1} \cdot\left(T_{2} \cdot T_{3}\right)$.

Lemma $3 T_{1} \cdot T_{2} \cdot T_{3} \cdots T_{n} \equiv\left(\left(\left(T_{1} \cdot T_{2}\right) \cdot T_{3}\right) \cdots\right) \cdot T_{n}$

Proof Since the composition is commutative and associative, we can build the entire system incrementally by composing two tasks at a time.
Lemma 4 The outgoing transitions from a given state represent every possible rendezvous that can occur at that particular state.

Proof According to the definition of $\delta$, we add an outgoing edge to a state for every rendezvous that can happen immediately after that state.

Multiple outgoing arcs from a state may represent choices due to control statements (such as if or while). $\delta\left(p_{1}, a\right)=q_{2}$ and $\delta\left(p_{1}, b\right)=q_{2}$, then we have two outgoing choices due to control flow.

On the other hand, a scheduling choice may occur when composing two tasks. A scheduling choice occurs when the ordering between two rendezvous is unknown. This happens when two different pairs of tasks can rendezvous on two different channels at the same time.

Suppose $a \in \Sigma_{1}$ and $a \notin \Sigma_{2}$ and $\delta_{1}\left(p_{1}, a\right)=q_{1}$, and if $b \in \Sigma_{2}$ and $b \notin \Sigma_{1}$ and $\delta_{2}\left(p_{2}, b\right)=q_{2}$, then $\delta_{12}\left(\left\langle p_{1}, p_{2}\right\rangle, a\right)=$ $\left\langle q_{1}, p_{2}\right\rangle$ and $\delta_{12}\left(\left\langle p_{1}, p_{2}\right\rangle, b\right)=\left\langle p_{1}, q_{2}\right\rangle$. Thus, for every possible scheduling choice, we have an outgoing edge from the given state.

The absence of any choice due to control or scheduling will leave it with either one or zero outgoing arcs. Consequently, the outgoing transitions from a given state represent all possible rendezvous that can occur at that particular state. They represent both control flow and scheduling choices.

A scheduling choice imposes no ordering among rendezvous, thus allowing the possibility of two or more rendezvous to happen at the same time.

Theorem 1 Two channels $a$ and $b$ can share buffers if, $\forall p$, at most one of $\delta(p, a)$ and $\delta(p, b)$ is defined, but not both.

Proof Suppose $a$ and $b$ can rendezvous at the same time and if $p_{1}$ represents the state of the program counter just before the rendezvous, then by Lemma 4 we have two outgoing arcs from $p_{1}: \delta\left(p_{1}, a\right)=q_{1}$ and $\delta\left(p_{1}, b\right)=q_{2}$

Consequently, for some $p$, both $\delta(p, a)$ and $\delta(p, b)$ exists. Conversely, if for all $p$ at most one of $\delta(p, a)$ and $\delta(p, b)$ exist, then we can safely say that $a$ and $b$ can share buffers.

Our algorithm does not differentiate between control flow choices (e.g., due to if or while) and scheduling choices (due to partial ordering of rendezvous). Both kinds of choices produce states having multiple outgoing arcs. We conclude that arcs going out from the same state cannot share buffers. The multiplicity can be contributed only by control choices leading to false positives, but our system is safe; whenever we are unsure, we do not allow sharing.

\section{Tackling State Space Explosion}

If two tasks communicate infrequently, there is a possibility that the number of states in the product machine will 
grow too large to deal with. We address this by introducing a threshold, which limits the stack depth of our recursive product machine composition procedure and corresponds to the longest simple path in the product machine. If we reach the threshold, we stop and treat the two tasks being composed as being separate (i.e., unable to share buffers between them).

This heuristic, which we chose because our implementation was running out of stack space on certain complex examples, has the advantage of applying exactly when we are unlikely to find opportunities to share buffer memory. Tightly coupled tasks tend to have small state spaces - these are exactly those that allow buffer memory to be shared. Loosely coupled tasks by definition run nearly independently and thus the communication pattern of most pairs of channels are uncontrolled, eliminating the chance to share buffers between them.

Algorithm 1 is the composition algorithm. It recursively composes two states $p_{1}$ and $p_{2}$. The depth variable is initialized to 0 and incremented whenever successor states are composed. Whenever depth exceeds the threshold, we declare failure.

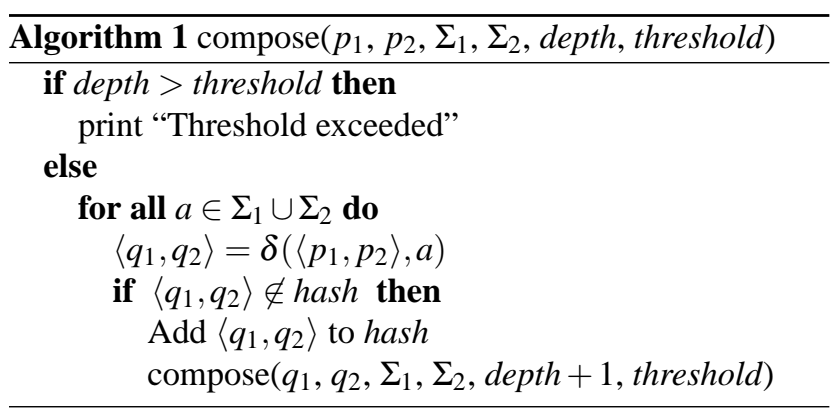

We draw conclusions about local channels (whose scope has been completely explored) and we remain silent about the others. We make safe conclusions even when other channels have not been completely explored.

Theorem 2 If our algorithm concludes that two channels a and $b$ can share buffers after abstracting away channel $c$, then $a$ and $b$ can still share buffers in the presence of $c$.

PROOF If $a$ and $b$ can share buffers, then there is a sequential ordering between them. By SHIM semantics [5], introduction of a new channel can create ordering between two channels that are not ordered, but can never disrupt an existing sequential ordering. Therefore, if our algorithm concludes that two buffers can share channels, introducing a new channel does not affect the conclusion.

We conclude that two channels can share buffers only if two conditions hold: the two channels have been explored completely and every state has at most one of the two channels in its outgoing edge set.
We take a bottom-up approach while merging groups of tasks. Tasks in a (preprocessed) SHIM program have a tree structure that arises from nesting of par constructs. We merge the leaf tasks of this tree before merging their parents. We stop merging when all tasks have exceeded the threshold or if the complete program has been merged. This approach allows us to stop whenever we run out of time or space without violating safety.

\section{Buffer Allocation}

Our static analysis algorithm produces a set $S$ that contains pairs of channels that can share buffers. Let $S^{\prime}$ be the complement of this set. We represent it as a graph: channels represent vertices and for every pair $\left\langle c_{i}, c_{j}\right\rangle \in S^{\prime}$, we draw an edge between $c_{i}$ and $c_{j}$. Two adjacent vertices cannot share buffers. Every node has a weight, which corresponds to the size of the channel.

Minimizing buffer memory consumption, therefore, reduces to the weighted vertex covering problem $[17, ?]$ : a graph $G$ is colored with $p$ colors such that no two adjacent vertices are of the same color. We denote the maximum weight of a vertex colored with color $i$ as $\max (i)$, and we need to find a coloring such that $\sum_{i=1}^{p} \max (i)$ is minimum. The problem is NP-hard.

We use a greedy first-fit algorithm to get an approximate solution. Let $G$ be a list of groups. Initially $G$ is empty. We order the channels in non-increasing order of buffer sizes, then add the channels one by one to the first non-conflicting group in $G$. If there is no such group, we create a new group in $G$ and add the channel to this newly created group. A group is defined to be non-conflicting if the channel to be added can share its buffer with every channel already in the group. Channels in the same group can share buffers. This algorithm runs in polynomial time but does not guarantee an optimal solution.

\section{Experimental Results}

We implemented our algorithm and ran it on various SHIM programs. Table 1 lists the results of running the experiments on a $3 \mathrm{GHz}$ Pentium 4 Linux machine with $1 \mathrm{~GB}$ RAM. For each example, the columns list the number of lines of code in the program, the total number of channels it uses, the number of tasks that take part in communication (i.e., excluding any functions that perform no communication), the number of bytes of buffer memory saved by applying our algorithm, what percentage this is of overall buffer memory, the time taken for analysis (including compilation, abstraction, verification, and grouping buffers), and the number of states our algorithm explored. For these experiments, we set the threshold to 8000 .

Source-Sink is a simple example of a FIFO with two processes: one that passes data and the other that prints the results through an output channel. Pipeline is a modification 


\begin{tabular}{lrrrrrrr}
\hline \multicolumn{1}{c}{ Example } & Lines & Channels & Tasks & Bytes Saved & Buffer Reduction & Runtime & States \\
\hline Source-Sink & 35 & 2 & 11 & 4 & $50 \%$ & $0.1 \mathrm{~s}$ & 394 \\
Pipeline & 35 & 5 & 9 & 16388 & 25 & 0.1 & 68 \\
Bitonic Sort & 35 & 5 & 13 & 12 & 60 & 0.1 & 135 \\
Prime Number Sieve & 40 & 5 & 16 & 12 & 60 & 0.5 & 122 \\
Berkeley & 40 & 3 & 11 & 4 & 33.33 & 0.6 & 285 \\
FIR Filter & 110 & 28 & 28 & 52 & 46.43 & 13.8 & 74646 \\
Framebuffer & 185 & 11 & 16 & 28 & 0.002 & 1.3 & 15761 \\
FFT & 230 & 14 & 15 & 344068 & 50 & 0.6 & 3750 \\
JPEG Decoder & 1020 & 7 & 15 & 772 & 50.13 & 1.8 & 517 \\
\hline
\end{tabular}

Table 1. Experimental results with the threshold set to 8000

\begin{tabular}{ccccc}
\hline Threshold & Bytes Saved & Buffer Reduction & Runtime & States \\
\hline 2000 & 0 & $0 \%$ & $0.6 \mathrm{~s}$ & 10024 \\
3000 & 0 & 0 & 1.5 & 23530 \\
4000 & 0 & 0 & 3.4 & 51086 \\
5000 & 52 & 46.43 & 12.4 & 70929 \\
6000 & 52 & 46.43 & 12.8 & 72101 \\
7000 & 52 & 46.43 & 13.5 & 73433 \\
8000 & 52 & 46.43 & 13.8 & 74646 \\
\hline
\end{tabular}

Table 2. Effect of threshold on the FIR filter example

of source-sink that uses two buffer processes in between the input and output process.

Bitonic Sort uses multiple tasks for that compare and shuffle pairs of data values. They interact through thirteen channels.

The Prime Number Sieve example has bounded recursion and uses the technique of [7] to remove it.

The Berkeley example has communication patterns that are data dependent. We abstract away the data, making it simpler to analyze.

Framebuffer contains a line drawing task that drives a $640 \times 480$ video framebuffer. The communication pattern is complicated.

FFT takes an audio file as input, divides it into 1024sample blocks performs fixed-point FFT on each block, then does an inverse FFT. It uses the largest buffers of all the example programs.

The JPEG decoder is one of the largest applications currently written in SHIM. It has multiple IDCT processors that run concurrently on groups of macroblocks passed around through buffers.

The FIR filter is a parallel filter with twenty-eight channels. It takes about thirteen seconds to analyze this program and the number of states explored is about eighty thousand. Since this was one of the more challenging examples for our algorithm, we tried varying the threshold. Table 2 summarizes our results. As expected, the number of visited states increases as we increase the threshold. With a threshold of 1000, we hardly explore the program, but higher thresholds let us explore more. When the threshold reaches 5000, we have explored enough of the system to begin to find opportunities for sharing buffer memory, even though we have not explored the system completely.

Experimentally, we find that the analysis takes less than a minute for modestly large programs and that we can reduce buffer space by $60 \%$ and therefore considerable amount of PPE memory on the Cell processor for examples like the bitonic sort and the prime number sieve.

\section{Related Work}

Many memory reduction techniques exist for embedded systems. Greef et al. [2] reduce array storage in a sequential program by reusing memory. Their approach has two phases: they internally reduce storage for each array, then globally try to share arrays. By contrast, our approach looks for sharing opportunities globally on communication buffers in a concurrent setting.

StreamIt [27] is a deterministic language like SHIM. Sermulins et al. [22] present cache aware optimizations that exploit communication pattern in StreamIt programs. They aim to improve instruction and data locality at the cost of data buffer size. Instead, we try to reduce buffer sizes.

Chrobak et al. [1] schedule tasks in a multiprocessor environment to minimize maximum buffer size. Our algo- 
rithm does not add scheduling constraints to the problem: it reduces the total buffer size without affecting the schedule, and thereby not affecting the overall speed.

The techniques of Murthy et al. [18, 19, 20, 21], Teich et al. [26], and Geilen et al. [8] are closest to ours. They describe several algorithms for merging buffers in signal processing systems that use synchronous data flow models [14]. Govindarajan et al. [9] minimize buffer space while executing at the optimal computation rate in dataflow networks. They cast this as a linear programming problem. Sofronis et al. [23] propose an optimal buffer scheme with a synchronous task model as basis. These papers revolve around minimizing buffers in a synchronous setting; our work solves similar problems in an asynchronous setting. Our approach finds if there is an ordering between rendezvous of different channels based on the product machine. We believe that our algorithm works on a richer set of programs.

Lin $[15,16]$ talks about an efficient compilation process of programs that have communication constructs similar to SHIM. He uses Petri nets to model the program and uses loop unrolling techniques. We did not attempt this approach because loop unrolling would cause the state space to explode even for small SHIM programs.

Static verification methods already exist for SHIM. In our previous work [28], we build a synchronous system to find deadlocks in a SHIM program. We make use of the fact that for a particular input sequence, if a SHIM program deadlocks under one schedule it will deadlock under any other. By contrast, the property we check in this paper is not schedule-independent: two channels may rendezvous at the same time under one schedule but may not under another schedule. This makes our problem more challenging.

There is a partial evaluation method [4] for SHIM that combines multiple concurrent processes to produce sequential code. Again, the work makes use of the scheduling independence property by expanding one task at a time until it terminates or blocks on a channel. On the other hand, in this paper, we expand all possible communications from a given state and therefore forcing us to consider all tasks that can communicate from that state, rather than a single task.

\section{Conclusions}

We presented a static buffer memory minimization technique for the SHIM concurrent language. We obtain the partial order between communication events on channels by forming the product machine of potentially all tasks in a program. To avoid state space explosion, we can treat the program as consisting of separate pieces.

We remove bounded recursion and expand each SHIM program into a tree of tasks and use sound abstractions to construct for each task an automaton that performs communication. Then we use the merging rules to combine tasks.
We abstract away data and computation from the program and only maintain parallel, communication and branch structures. We abstract away the data-dependent decisions formed by conditionals and loops and do not differentiate between scheduling choices and conditional branches. This may lead to false positives: our technique can discard pairs even though they can share buffers. However, our experimental results suggest this is not a big disadvantage and in any case our technique remains safe.

Our algorithm can be practically applied to the SHIM compiler that generates code for the Cell Broadband Engine. We found we could save 344KB of the PPE's memory for an FFT example.

We reduce memory without affecting the run-time schedule or performance. By sharing, two or more buffer pointers point to the same memory location. This can be done at compile-time during the code-generation phase.

To avoid state space explosion, we introduced a threshold for limiting the recursion depth our algorithm must handle. We plan to look into more modular techniques that allow a set of tasks to be analyzed independently of the remaining sets.

We currently ignore SHIM's exceptions [25]. Exceptions in SHIM provide a convenient way to terminate peer tasks and they are deterministic in behavior. We also plan to consider exceptions in the future.

\section{Acknowledgements}

This work was supported by NSF grant CNS-0720292.

\section{References}

[1] M. Chrobak, J. Csirik, C. Imreh, J. Noga, J. Sgall, and G. J. Woeginger. The buffer minimization problem for multiprocessor scheduling with conflicts. In ICALP '01: Proceedings of the 28th International Colloquium on Automata, Languages and Programming, pages 862-874, London, UK, 2001. Springer-Verlag.

[2] E. de Greef, F. Catthoor, and H. de Man. Array placement for storage size reduction in embedded multimedia systems. In ASAP '97: Proceedings of the IEEE International Conference on Application-Specific Systems, Architectures and Processors, page 66, Washington, DC, USA, 1997. IEEE Computer Society.

[3] S. A. Edwards and O. Tardieu. SHIM: A deterministic model for heterogeneous embedded systems. In Proceedings of the International Conference on Embedded Software (Emsoft), pages 37-44, Jersey City, New Jersey, Sept. 2005.

[4] S. A. Edwards and O. Tardieu. Efficient code generation from SHIM models. In Proceedings of Languages, Compilers, and Tools for Embedded Systems (LCTES), pages 125134, Ottawa, Canada, June 2006.

[5] S. A. Edwards and O. Tardieu. SHIM: A deterministic model for heterogeneous embedded systems. IEEE Transactions on Very Large Scale Integration (VLSI) Systems, 14(8):854867, Aug. 2006. 
[6] S. A. Edwards, N. Vasudevan, and O. Tardieu. Programming shared memory multiprocessors with deterministic message-passing concurrency: Compiling SHIM to Pthreads. In Proceedings of Design, Automation, and Test in Europe (DATE), pages 1498-1503, Munich, Germany, Mar. 2008.

[7] S. A. Edwards and J. Zeng. Static elaboration of recursion for concurrent software. In Proceedings of the Workshop on Partial Evaluation and Program Manipulation (PEPM), San Francisco, California, Jan. 2008.

[8] M. Geilen, T. Basten, and S. Stuijk. Minimising buffer requirements of synchronous dataflow graphs with model checking. In DAC '05: Proceedings of the 42nd annual conference on Design automation, pages 819-824, New York, NY, USA, 2005. ACM.

[9] R. Govindarajan, G. R. Gao, and P. D. Y. Minimizing buffer requirements under rate-optimal schedule in regular dataflow networks. Journal of VLSI Signal Processing Systems, 31(3):207-209, July 2002.

[10] C. A. R. Hoare. Communicating Sequential Processes. Prentice Hall, Upper Saddle River, New Jersey, 1985.

[11] J. A. Kahle, M. N. Day, H. P. Hofstee, C. R. Johns, T. R. Maeurer, and D. Shippy. Introduction to the Cell multiprocessor. IBM Journal of Research and Development, 49(4/5):589-604, July/September 2005.

[12] G. Kahn. The semantics of a simple language for parallel programming. In Information Processing 74: Proceedings of IFIP Congress 74, pages 471-475, Stockholm, Sweden, Aug. 1974. North-Holland.

[13] M. Kistler, M. Perrone, and F. Petrini. Cell multiprocessor communication network: Built for speed. IEEE Micro, 26(3):10-23, May-June 2006.

[14] E. A. Lee and D. G. Messerschmitt. Synchronous data flow. Proceedings of the IEEE, 75(9):1235-1245, Sept. 1987.

[15] B. Lin. Efficient compilation of process-based concurrent programs without run-time scheduling. In Proceedings of Design, Automation, and Test in Europe (DATE), pages 211217, Paris, France, Feb. 1998.

[16] B. Lin. Software synthesis of process-based concurrent programs. In Proceedings of the 35th Design Automation Conference, pages 502-505, San Francisco, California, June 1998.

[17] E. Malaguti, M. Monaci, and P. Toth. Models and heuristic algorithms for a weighted vertex coloring problem. Journal of Heuristics, 2008.

[18] P. K. Murthy and S. S. Bhattacharyya. Systematic consolidation of input and output buffers in synchronous dataflow specifications. IEEE Workshop on Signal Processing Systems (SiPS), pages 673-682, 2000.

[19] P. K. Murthy and S. S. Bhattacharyya. Shared buffer implementations of signal processing systems using lifetime analysis techniques. IEEE Transactions on Computer-Aided
Design of Integrated Circuits and Systems, 20(2):177-198, Feb. 2001.

[20] P. K. Murthy and S. S. Bhattacharyya. Buffer merginga powerful technique for reducing memory requirements of synchronous dataflow specifications. ACM Transactions on Design Automation of Electronic Systems, 9(2):212-237, Apr. 2004.

[21] P. K. Murthy and S. S. Bhattacharyya. Memory Management for Synthesis of DSP Software. CRC Press, Inc., Boca Raton, FL, USA, 2006.

[22] J. Sermulins, W. Thies, R. Rabbah, and S. Amarasinghe. Cache aware optimization of stream programs. In Proceedings of Languages, Compilers, and Tools for Embedded Systems (LCTES), pages 115-126, New York, NY, USA, 2005. ACM.

[23] C. Sofronis, S. Tripakis, and P. Caspi. A memory-optimal buffering protocol for preservation of synchronous semantics under preemptive scheduling. In Proceedings of the International Conference on Embedded Software (Emsoft), pages 21-33, New York, NY, USA, 2006. ACM.

[24] O. Tardieu and S. A. Edwards. R-SHIM: Deterministic concurrency with recursion and shared variables. In Proceedings of the International Conference on Formal Methods and Models for Codesign (MEMOCODE), page 202, Napa, California, July 2006.

[25] O. Tardieu and S. A. Edwards. Scheduling-independent threads and exceptions in SHIM. In Proceedings of the International Conference on Embedded Software (Emsoft), pages 142-151, Seoul, Korea, Oct. 2006.

[26] J. Teich, E. Zitzler, and S. S. Bhattacharyya. Buffer memory optimization in dsp applications - an evolutionary approach. In Proceedings of Parallel Problem Solving from Nature (PPSN), pages 885-896, London, UK, 1998. Springer-Verlag.

[27] W. Thies, M. Karczmarek, and S. Amarasinghe. StreamIt: A language for streaming applications. In Proceedings of the International Conference on Compiler Construction (CC), volume 2304 of Lecture Notes in Computer Science, pages 179-196, Grenoble, France, Apr. 2002.

[28] N. Vasudevan and S. A. Edwards. Static deadlock detection for the SHIM concurrent language. In Proceedings of the International Conference on Formal Methods and Models for Codesign (MEMOCODE), Anaheim, California, June 2008.

[29] N. Vasudevan and S. A. Edwards. Celling SHIM: Compiling deterministic concurrency to a heterogeneous multicore. In Proceedings of the Symposium on Applied Computing (SAC), volume III, pages 1626-1631, Honolulu, Hawaii, Mar. 2009.

[30] N. Vasudevan, S. Singh, and S. A. Edwards. A deterministic multi-way rendezvous library for Haskell. In Proceedings of the International Parallel and Distributed Processing Symposium (IPDPS), Miami, Florida, Apr. 2008. 